Acta Crystallographica Section E

Structure Reports

Online

ISSN 1600-5368

\section{2-(4-Chlorophenyl)-3-methyl-N-(5- methylthiazol-2-yl)butanamide}

\section{Jing-Li Cheng, Jin-Hao Zhao, ${ }^{a}$ Guo-Nian Zhu ${ }^{a}$ and Fu-Cheng $\operatorname{Lin}^{\mathrm{b} *}$}

${ }^{a}$ College of Agriculture and Biotechnology, Zhejiang University, Hangzhou 310029, People's Republic of China, and ' Institute of Biotechnology, Zhejiang University, Hangzhou 310029, People's Republic of China

Correspondence e-mail: fuchenglin@zj.com

Received 19 November 2008; accepted 17 December 2008

Key indicators: single-crystal X-ray study; $T=298 \mathrm{~K}$; mean $\sigma(\mathrm{C}-\mathrm{C})=0.003 \AA$; $R$ factor $=0.040 ; w R$ factor $=0.172 ;$ data-to-parameter ratio $=20.3$.

In the title compound, $\mathrm{C}_{15} \mathrm{H}_{17} \mathrm{ClN}_{2} \mathrm{OS}$, the thiazole ring, which is essentially planar with a maximum deviation of 0.044 (3) $\AA$, makes a dihedral angle of $54.76(8)^{\circ}$ with the benzene ring. In the crystal, adjacent molecules related by twofold rotation symmetry are linked by pairs of $\mathrm{N}-\mathrm{H} \cdots \mathrm{N}$ hydrogen bonds.

\section{Related literature}

For background, see: Holmstead et al. (1978); Forlani (1978). For a related structure, see: Zhao et al. (2006).

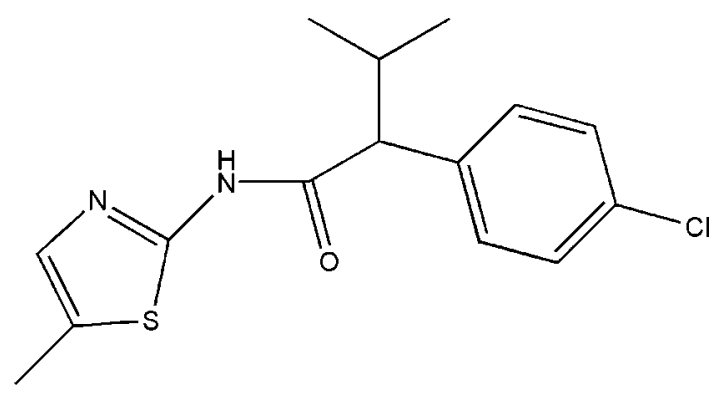

\section{Experimental}

Crystal data

$\mathrm{C}_{15} \mathrm{H}_{17} \mathrm{ClN}_{2} \mathrm{OS}$

$M_{r}=308.83$

Monoclinic, $C 2 / c$

$a=14.9649$ (6) §

$$
\begin{aligned}
& Z=8 \\
& \text { Mo } K \alpha \text { radiation } \\
& \mu=0.36 \mathrm{~mm}^{-1}
\end{aligned}
$$

Data collection

Rigaku R-AXIS RAPID

diffractometer

Absorption correction: multi-scan (ABSCOR; Higashi, 1995)

$T_{\min }=0.858, T_{\max }=0.911$

Refinement

$R\left[F^{2}>2 \sigma\left(F^{2}\right)\right]=0.040$

$w R\left(F^{2}\right)=0.172$

$S=1.01$

3708 reflections

$T=298(1) \mathrm{K}$

$0.41 \times 0.33 \times 0.26 \mathrm{~mm}$

15655 measured reflections 3708 independent reflections 2559 reflections with $F^{2}>2 \sigma\left(F^{2}\right)$ $R_{\text {int }}=0.027$

Table 1

Hydrogen-bond geometry $\left(\AA{ }^{\circ}\right)$.

\begin{tabular}{lllll}
\hline$D-\mathrm{H} \cdots A$ & $D-\mathrm{H}$ & $\mathrm{H} \cdots A$ & $D \cdots A$ & $D-\mathrm{H} \cdots A$ \\
\hline $\mathrm{N} 1-\mathrm{H} 111 \cdots \mathrm{N} 2^{\mathrm{i}}$ & 0.86 & 2.08 & $2.929(2)$ & 168 \\
\hline
\end{tabular}

Symmetry code: (i) $-x+1, y,-z+\frac{3}{2}$.

Data collection: PROCESS-AUTO (Rigaku, 1998); cell refinement: PROCESS-AUTO; data reduction: CrystalStructure (Rigaku/ MSC, 2004); program(s) used to solve structure: SIR97 (Altomare et al., 1999); program(s) used to refine structure: SHELXL97 (Sheldrick, 2008); molecular graphics: ORTEP-3 (Farrugia, 1997); software used to prepare material for publication: CrystalStructure.

The authors are grateful for support from the National Natural Science Foundation of China (No. 30700532) and also thank Professor Jian-Ming Gu for help with the analysis of the crystal data.

Supplementary data and figures for this paper are available from the IUCr electronic archives (Reference: IS2368).

\section{References}

Altomare, A., Burla, M. C., Camalli, M., Cascarano, G. L., Giacovazzo, C., Guagliardi, A., Moliterni, A. G. G., Polidori, G. \& Spagna, R. (1999). J. Appl. Cryst. 32, 115-119.

Farrugia, L. J. (1997). J. Appl. Cryst. 30, 565.

Forlani, L. (1978). J. Chem. Soc. Perkin Trans. 1, pp. 1169-1171.

Higashi, T. (1995). ABSCOR. Rigaku Corporation, Tokyo, Japan.

Holmstead, R. L., Fullmer, D. G. \& Ruzo, L. O. (1978). J. Agric. Food Chem. 26, 954-959.

Rigaku (1998). PROCESS-AUTO. Rigaku Corporation, Tokyo, Japan.

Rigaku/MSC (2004). CrystalStructure. Rigaku/MSC, The Woodlands, Texas, USA.

Sheldrick, G. M. (2008). Acta Cryst. A64, 112-122.

Zhao, J.-H., Cheng, J.-L., Huang, Y.-K. \& Zhu, G.-N. (2006). Acta Cryst. E62, o4840-o4841. 


\section{supporting information}

Acta Cryst. (2009). E65, o184 [doi:10.1107/S1600536808043031]

\section{2-(4-Chlorophenyl)-3-methyl-N-(5-methylthiazol-2-yl)butanamide}

\section{Jing-Li Cheng, Jin-Hao Zhao, Guo-Nian Zhu and Fu-Cheng Lin}

\section{S1. Comment}

2-(4-Chlorophenyl)-3-methylbutanoyl chloride is an intermediate in the synthesis of fenvalerate, an excellent insecticide (Holmstead et al., 1978). 2-Amino-5-methyl-thiazole is another heterocyclic intermediate (Forlani, 1978). As part of our continuing interest in the design and synthesis of new pesticides, we have isolated the title compound, (I), the product of the condensation reaction between 2-(4-chlorophenyl)-3-methylbutanoyl chloride and 5-methyl-2-aminothiazole, as colourless crystals suitable for X-ray analysis.

The molecular structure of (I) is illustrated in Fig. 1. Atoms N2, C10, C11, S1, C9 and N1 are coplanar, the largest deviation being 0.044 (3) $\AA$ for N1. As expected, the benzene ring is planar, and atom Cl1 lies only 0.018 (4) $\AA$ from the plane defined by the ring $\mathrm{C}$ atoms and itself. The angle between these two rings is $54.76(8)^{\circ}$, smaller than the angle between the thiazole and benzene rings of the compound 2-(4-chlorophenyl)-3-methyl- $N$-(thiazol-2-yl) butanamide (Zhao et al., 2006). There are $\mathrm{N}-\mathrm{H} \cdots \mathrm{N}$ interactions in the crystal structure, which lead to the formation of hydrogen-bonded dimers (Figs. 2 and 3).

\section{S2. Experimental}

2-Amino-5-methylthiazole $(1.14 \mathrm{~g}, 10 \mathrm{mmol})$, 4-dimethylaminopyridine $(0.12 \mathrm{~g})$, triethylamine $(1.31 \mathrm{~g})$ and chloroform $(100 \mathrm{ml})$ were added to a $250 \mathrm{ml}$ round flask. The mixture was stirred and cooled to $273 \mathrm{~K}$, and then 2-(4-chlorophenyl)-3-methylbutanoyl chloride (3.47 g) was added dropwise within $30 \mathrm{~min}$. The mixture was stirred at room temperature for $3 \mathrm{~h}$ and then $1 \%$ aqueous $\mathrm{HCl}$ was added $(5 \mathrm{ml})$. The organic layer was washed with water to a neutral $\mathrm{pH}$ and dried over $\mathrm{Na}_{2} \mathrm{SO}_{4}$. After being filtered and concentrated, the organic residue was purified by silica-gel column chromatography, eluted with ethyl acetate-petroleum ether-formic acid $(10: 80: 1, v / v / v)$, to give a white solid (yield 85\%, $2.5 \mathrm{~g})$, (I). It was then recrystallized from ethyl acetate-petroleum ether $(2: 1, v / v)$ to give colourless blocks (m.p. 460-461 $\mathrm{K})$.

\section{S3. Refinement}

$\mathrm{H}$ atoms were included in calculated positions and refined using a riding model, with $\mathrm{C}-\mathrm{H}$ distances constrained to 0.96 $\AA$ for methyl $\mathrm{H}$ atoms, $0.93 \AA$ for aryl $\mathrm{H}$ atoms and $0.98 \AA$ for the remainder, with $\mathrm{N}-\mathrm{H}$ distances constrained to $0.86 \AA$, and with $U_{\text {iso }}(\mathrm{H})=1.2 U_{\text {eq }}(\mathrm{C}, \mathrm{N})$ or $1.5 U_{\text {eq }}($ methyl C). 


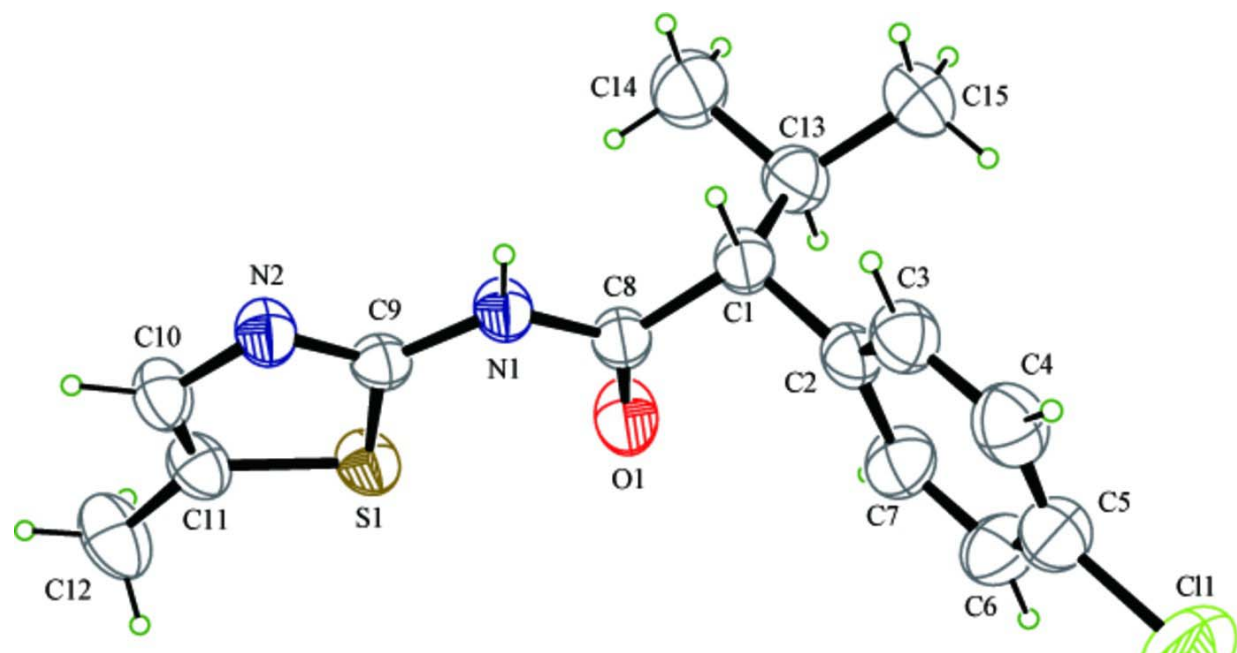

\section{Figure 1}

The molecular structure of (I), showing the atom-labelling scheme. Displacement ellipsoids are drawn at the 30\% probability level.

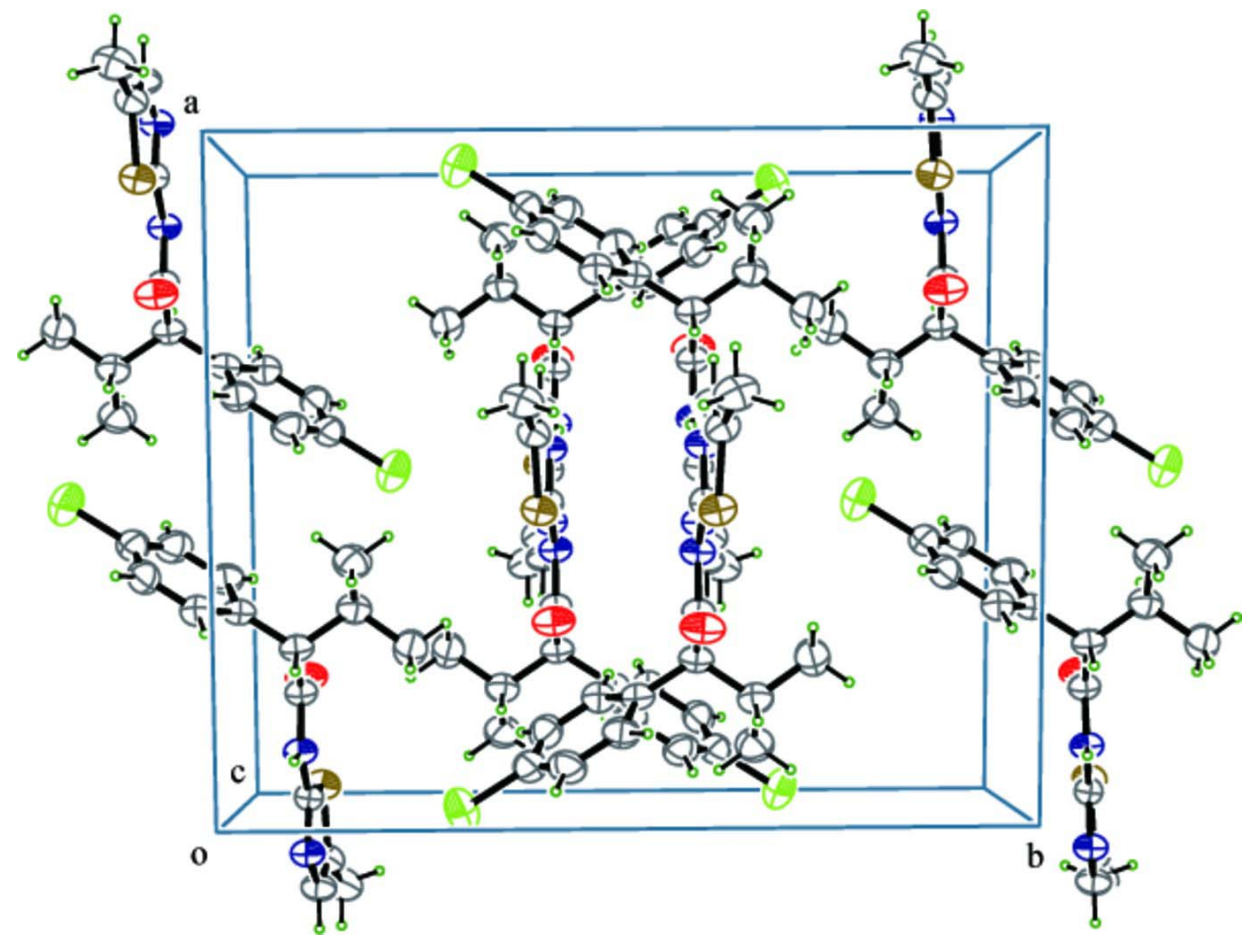

Figure 2

Molecular packing arrangement in the unit cell. 


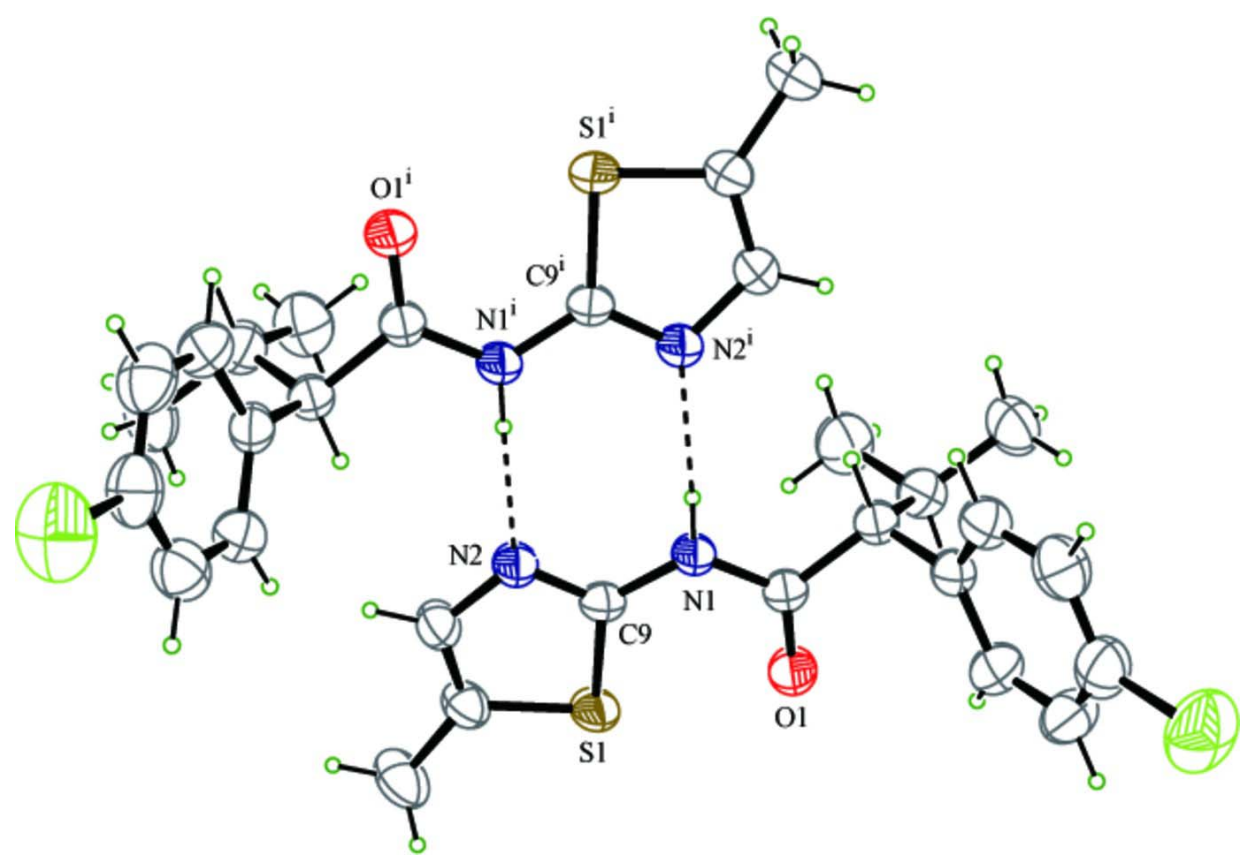

\section{Figure 3}

View showing the $\mathrm{N}-\mathrm{H}^{\cdots} \mathrm{N}$ hydrogen bonding (dashed lines) [symmetry code: (i) $\left.1-x, y, 3 / 2-z\right]$.

\section{2-(4-Chlorophenyl)-3-methyl-N-(5-methylthiazol-2-yl)butanamide}

Crystal data

$\mathrm{C}_{15} \mathrm{H}_{17} \mathrm{ClN}_{2} \mathrm{OS}$

$M_{r}=308.83$

Monoclinic, $C 2 / c$

Hall symbol: $-\mathrm{C} 2 \mathrm{yc}$

$a=14.9649(6) \AA$

$b=17.6062(7) \AA$

$c=12.5606(5) \AA$

$\beta=99.9482(11)^{\circ}$

$V=3259.6(2) \AA^{3}$

$Z=8$

Data collection

Rigaku R-AXIS RAPID

diffractometer

Detector resolution: 10.00 pixels $\mathrm{mm}^{-1}$

$\omega$ scans

Absorption correction: multi-scan

(ABSCOR; Higashi, 1995)

$T_{\min }=0.858, T_{\max }=0.911$

15655 measured reflections

\section{Refinement}

Refinement on $F^{2}$

$R\left[F^{2}>2 \sigma\left(F^{2}\right)\right]=0.040$

$w R\left(F^{2}\right)=0.172$

$S=1.01$

3708 reflections
$F(000)=1296.00$

$D_{\mathrm{x}}=1.258 \mathrm{Mg} \mathrm{m}^{-3}$

Mo $K \alpha$ radiation, $\lambda=0.71075 \AA$

Cell parameters from 10539 reflections

$\theta=3.3-27.4^{\circ}$

$\mu=0.36 \mathrm{~mm}^{-1}$

$T=298 \mathrm{~K}$

Block, colorless

$0.41 \times 0.33 \times 0.26 \mathrm{~mm}$

3708 independent reflections

2559 reflections with $F^{2}>2 \sigma\left(F^{2}\right)$

$R_{\text {int }}=0.027$

$\theta_{\text {max }}=27.4^{\circ}$

$h=-19 \rightarrow 19$

$k=-22 \rightarrow 22$

$l=-16 \rightarrow 15$

183 parameters

0 restraints

$\mathrm{H}$-atom parameters constrained

$w=1 /\left[\sigma^{2}\left(F_{\mathrm{o}}^{2}\right)+(0.121 P)^{2}\right]$

where $P=\left(F_{\mathrm{o}}{ }^{2}+2 F_{\mathrm{c}}{ }^{2}\right) / 3$ 
$(\Delta / \sigma)_{\max }=0.001$

$\Delta \rho_{\max }=0.27 \mathrm{e} \AA^{-3}$

$\Delta \rho_{\min }=-0.29$ e $\AA^{-3}$

Special details

Geometry. ENTER SPECIAL DETAILS OF THE MOLECULAR GEOMETRY

Refinement. Refinement using all reflections. The weighted $R$-factor $(w R)$ and goodness of fit $(S)$ are based on $F^{2} . R$ factor (gt) are based on $F$. The threshold expression of $F^{2}>2.0 \sigma\left(F^{2}\right)$ is used only for calculating $R$-factor (gt).

Fractional atomic coordinates and isotropic or equivalent isotropic displacement parameters $\left(\AA^{2}\right)$

\begin{tabular}{|c|c|c|c|c|}
\hline & $x$ & $y$ & $z$ & $U_{\text {iso }} * / U_{\text {eq }}$ \\
\hline $\mathrm{Cl1}$ & $0.02408(6)$ & $0.70387(4)$ & $0.59960(9)$ & $0.1206(3)$ \\
\hline $\mathrm{S} 1$ & $0.46203(3)$ & $0.39223(3)$ & $0.44117(3)$ & 0.0559 (2) \\
\hline $\mathrm{O} 1$ & $0.29216(10)$ & $0.40983(10)$ & $0.48366(10)$ & $0.0696(4)$ \\
\hline N1 & $0.40090(10)$ & $0.41256(9)$ & $0.63283(11)$ & $0.0496(3)$ \\
\hline N2 & $0.55713(11)$ & $0.40575(9)$ & $0.63079(12)$ & $0.0530(4)$ \\
\hline $\mathrm{C} 1$ & $0.24241(12)$ & $0.41401(12)$ & $0.65622(13)$ & $0.0534(4)$ \\
\hline $\mathrm{C} 2$ & $0.18766(12)$ & $0.48677(12)$ & $0.64116(13)$ & $0.0531(4)$ \\
\hline $\mathrm{C} 3$ & $0.18371(13)$ & $0.53424(12)$ & $0.72811(17)$ & $0.0611(5)$ \\
\hline $\mathrm{C} 4$ & $0.13313(17)$ & $0.60024(12)$ & $0.7171(2)$ & $0.0739(6)$ \\
\hline $\mathrm{C} 5$ & $0.08679(16)$ & $0.61988(13)$ & $0.6158(2)$ & $0.0756(6)$ \\
\hline C6 & $0.08973(17)$ & $0.57396(14)$ & $0.5282(2)$ & $0.0790(6)$ \\
\hline $\mathrm{C} 7$ & $0.13902(13)$ & $0.50774(13)$ & $0.54031(16)$ & $0.0667(5)$ \\
\hline $\mathrm{C} 8$ & $0.31263(12)$ & $0.41250(11)$ & $0.58189(13)$ & $0.0506(4)$ \\
\hline C9 & $0.47375(12)$ & $0.40501(10)$ & $0.57886(13)$ & $0.0452(4)$ \\
\hline $\mathrm{C} 10$ & $0.61571(13)$ & $0.39512(12)$ & $0.55850(16)$ & $0.0583(5)$ \\
\hline C11 & $0.57888(13)$ & $0.38625(12)$ & $0.45425(16)$ & $0.0563(5)$ \\
\hline $\mathrm{C} 12$ & $0.62499(18)$ & $0.37260(17)$ & 0.35901 (19) & $0.0818(7)$ \\
\hline $\mathrm{C} 13$ & $0.18427(14)$ & $0.34156(12)$ & $0.63811(17)$ & $0.0646(5)$ \\
\hline $\mathrm{C} 14$ & $0.24379(18)$ & $0.27066(14)$ & $0.6583(2)$ & $0.0850(7)$ \\
\hline $\mathrm{C} 15$ & $0.11223(17)$ & $0.34038(16)$ & $0.7103(2)$ & $0.0856(7)$ \\
\hline H1 & 0.2751 & 0.4131 & 0.7309 & $0.064 *$ \\
\hline $\mathrm{H} 3$ & 0.2160 & 0.5213 & 0.7957 & $0.073 *$ \\
\hline $\mathrm{H} 4$ & 0.1303 & 0.6309 & 0.7766 & $0.089 *$ \\
\hline H6 & 0.0583 & 0.5877 & 0.4605 & $0.095 *$ \\
\hline H7 & 0.1400 & 0.4765 & 0.4808 & $0.080 *$ \\
\hline H10 & 0.6782 & 0.3942 & 0.5812 & $0.070 *$ \\
\hline H13 & 0.1535 & 0.3409 & 0.5626 & $0.078^{*}$ \\
\hline H111 & 0.4119 & 0.4175 & 0.7020 & $0.060 *$ \\
\hline H121 & 0.6896 & 0.3732 & 0.3823 & $0.098^{*}$ \\
\hline H122 & 0.6081 & 0.4118 & 0.3062 & $0.098^{*}$ \\
\hline H123 & 0.6068 & 0.3241 & 0.3276 & $0.098 *$ \\
\hline H141 & 0.2729 & 0.2694 & 0.7326 & $0.102 *$ \\
\hline H142 & 0.2890 & 0.2719 & 0.6126 & $0.102 *$ \\
\hline H143 & 0.2068 & 0.2262 & 0.6422 & $0.102 *$ \\
\hline H151 & 0.0750 & 0.2960 & 0.6946 & $0.103^{*}$ \\
\hline H152 & 0.0752 & 0.3850 & 0.6972 & $0.103^{*}$ \\
\hline
\end{tabular}

Extinction coefficient: 0.0028 (7)
Extinction correction: SHELXL 
supporting information

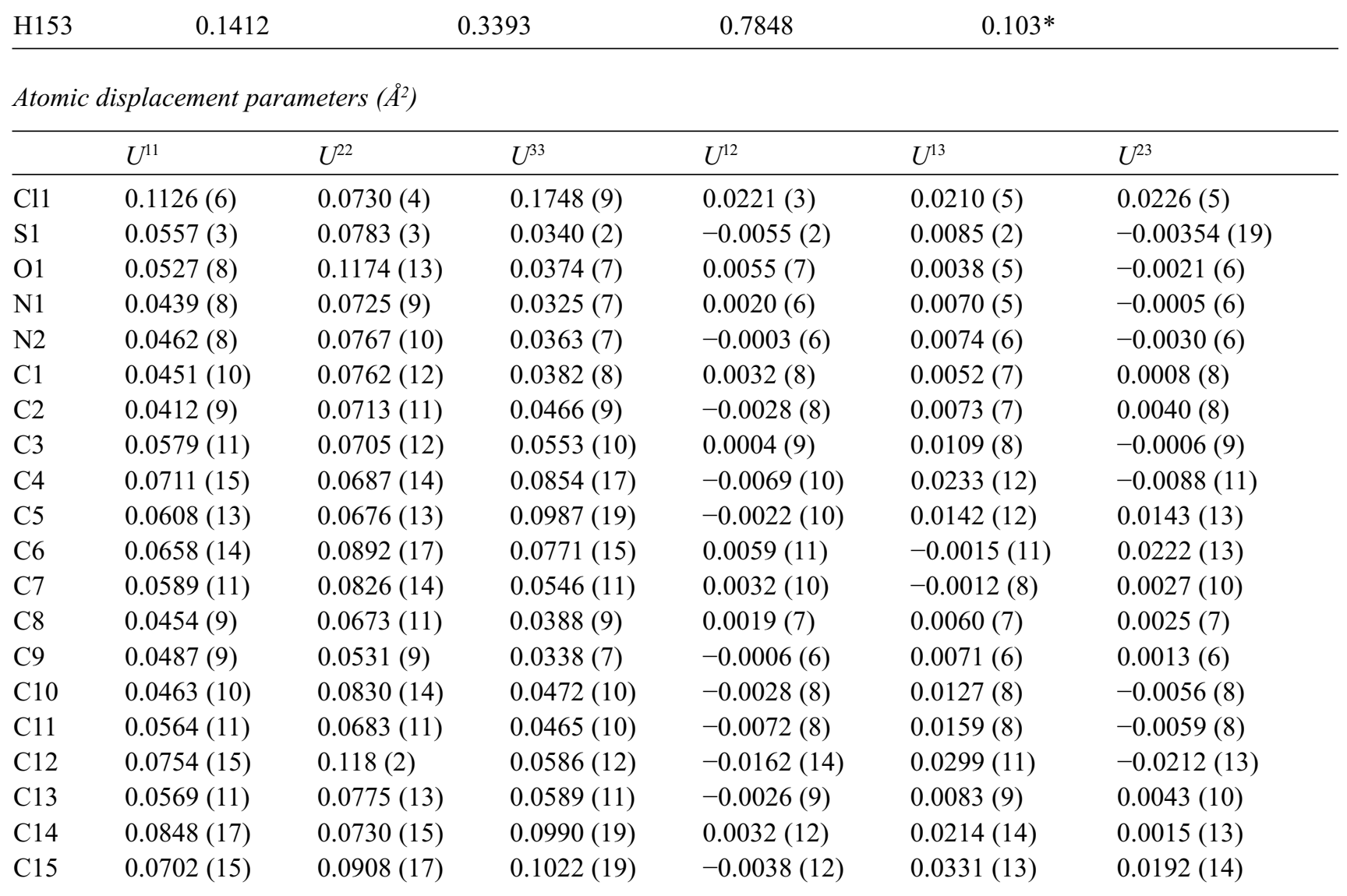

Geometric parameters $\left(\AA,{ }^{\circ}\right)$

\begin{tabular}{llll}
\hline $\mathrm{C} 11-\mathrm{C} 5$ & $1.744(2)$ & $\mathrm{C} 13-\mathrm{C} 14$ & $1.529(3)$ \\
$\mathrm{S} 1-\mathrm{C} 9$ & $1.7227(17)$ & $\mathrm{C} 13-\mathrm{C} 15$ & $1.524(3)$ \\
$\mathrm{S} 1-\mathrm{C} 11$ & $1.731(2)$ & $\mathrm{N} 1-\mathrm{H} 111$ & 0.860 \\
$\mathrm{O} 1-\mathrm{C} 8$ & $1.220(2)$ & $\mathrm{C} 1-\mathrm{H} 1$ & 0.980 \\
$\mathrm{~N} 1-\mathrm{C} 8$ & $1.365(2)$ & $\mathrm{C} 3-\mathrm{H} 3$ & 0.930 \\
$\mathrm{~N} 1-\mathrm{C} 9$ & $1.386(2)$ & $\mathrm{C} 4-\mathrm{H} 4$ & 0.930 \\
$\mathrm{~N} 2-\mathrm{C} 9$ & $\mathrm{C} 6-\mathrm{H} 6$ & 0.930 \\
$\mathrm{~N} 2-\mathrm{C} 10$ & $1.304(2)$ & $\mathrm{C} 7-\mathrm{H} 7$ & 0.930 \\
$\mathrm{C} 1-\mathrm{C} 2$ & $1.380(2)$ & $\mathrm{C} 10-\mathrm{H} 10$ & 0.930 \\
$\mathrm{C} 1-\mathrm{C} 8$ & $1.515(2)$ & $\mathrm{C} 12-\mathrm{H} 121$ & 0.960 \\
$\mathrm{C} 1-\mathrm{C} 13$ & $1.522(2)$ & $\mathrm{C} 12-\mathrm{H} 122$ & 0.960 \\
$\mathrm{C} 2-\mathrm{C} 3$ & $1.539(2)$ & $\mathrm{C} 12-\mathrm{H} 123$ & 0.960 \\
$\mathrm{C} 2-\mathrm{C} 7$ & $1.385(2)$ & $\mathrm{C} 13-\mathrm{H} 13$ & 0.980 \\
$\mathrm{C} 3-\mathrm{C} 4$ & $1.397(2)$ & $\mathrm{C} 14-\mathrm{H} 141$ & 0.960 \\
$\mathrm{C} 4-\mathrm{C} 5$ & $1.381(3)$ & $\mathrm{C} 14-\mathrm{H} 142$ & 0.960 \\
$\mathrm{C} 5-\mathrm{C} 6$ & $1.384(3)$ & $\mathrm{C} 15-\mathrm{H} 151$ & 0.960 \\
$\mathrm{C} 6-\mathrm{C} 7$ & $1.372(3)$ & $\mathrm{C} 15-\mathrm{H} 152$ & 0.960 \\
$\mathrm{C} 10-\mathrm{C} 11$ & $1.374(3)$ & $\mathrm{C} 15-\mathrm{H} 153$ & 0.960 \\
$\mathrm{C} 11-\mathrm{C} 12$ & $1.339(2)$ &
\end{tabular}




\begin{tabular}{|c|c|c|c|}
\hline $\mathrm{C} 9-\mathrm{S} 1-\mathrm{C} 11$ & $89.24(9)$ & $\mathrm{C} 13-\mathrm{C} 1-\mathrm{H} 1$ & 107.7 \\
\hline $\mathrm{C} 8-\mathrm{N} 1-\mathrm{C} 9$ & $123.37(14)$ & $\mathrm{C} 2-\mathrm{C} 3-\mathrm{H} 3$ & 119.1 \\
\hline $\mathrm{C} 9-\mathrm{N} 2-\mathrm{C} 10$ & $109.35(15)$ & $\mathrm{C} 4-\mathrm{C} 3-\mathrm{H} 3$ & 119.1 \\
\hline $\mathrm{C} 2-\mathrm{C} 1-\mathrm{C} 8$ & $110.74(16)$ & $\mathrm{C} 3-\mathrm{C} 4-\mathrm{H} 4$ & 120.6 \\
\hline $\mathrm{C} 2-\mathrm{C} 1-\mathrm{C} 13$ & $113.75(15)$ & $\mathrm{C} 5-\mathrm{C} 4-\mathrm{H} 4$ & 120.6 \\
\hline $\mathrm{C} 8-\mathrm{C} 1-\mathrm{C} 13$ & $109.12(16)$ & $\mathrm{C} 5-\mathrm{C} 6-\mathrm{H} 6$ & 119.9 \\
\hline $\mathrm{C} 1-\mathrm{C} 2-\mathrm{C} 3$ & $120.52(15)$ & $\mathrm{C} 7-\mathrm{C} 6-\mathrm{H} 6$ & 119.9 \\
\hline $\mathrm{C} 1-\mathrm{C} 2-\mathrm{C} 7$ & $121.50(17)$ & $\mathrm{C} 2-\mathrm{C} 7-\mathrm{H} 7$ & 119.7 \\
\hline $\mathrm{C} 3-\mathrm{C} 2-\mathrm{C} 7$ & $117.97(19)$ & $\mathrm{C} 6-\mathrm{C} 7-\mathrm{H} 7$ & 119.7 \\
\hline $\mathrm{C} 2-\mathrm{C} 3-\mathrm{C} 4$ & $121.75(19)$ & $\mathrm{N} 2-\mathrm{C} 10-\mathrm{H} 10$ & 121.4 \\
\hline $\mathrm{C} 3-\mathrm{C} 4-\mathrm{C} 5$ & $118.8(2)$ & $\mathrm{C} 11-\mathrm{C} 10-\mathrm{H} 10$ & 121.4 \\
\hline $\mathrm{C} 11-\mathrm{C} 5-\mathrm{C} 4$ & $119.5(2)$ & $\mathrm{C} 11-\mathrm{C} 12-\mathrm{H} 121$ & 109.5 \\
\hline $\mathrm{C} 11-\mathrm{C} 5-\mathrm{C} 6$ & $119.8(2)$ & $\mathrm{C} 11-\mathrm{C} 12-\mathrm{H} 122$ & 109.5 \\
\hline $\mathrm{C} 4-\mathrm{C} 5-\mathrm{C} 6$ & $120.6(2)$ & $\mathrm{C} 11-\mathrm{C} 12-\mathrm{H} 123$ & 109.5 \\
\hline $\mathrm{C} 5-\mathrm{C} 6-\mathrm{C} 7$ & $120.1(2)$ & $\mathrm{H} 121-\mathrm{C} 12-\mathrm{H} 122$ & 109.5 \\
\hline $\mathrm{C} 2-\mathrm{C} 7-\mathrm{C} 6$ & $120.7(2)$ & $\mathrm{H} 121-\mathrm{C} 12-\mathrm{H} 123$ & 109.5 \\
\hline $\mathrm{O} 1-\mathrm{C} 8-\mathrm{N} 1$ & $121.86(17)$ & $\mathrm{H} 122-\mathrm{C} 12-\mathrm{H} 123$ & 109.5 \\
\hline $\mathrm{O} 1-\mathrm{C} 8-\mathrm{C} 1$ & $122.81(15)$ & $\mathrm{C} 1-\mathrm{C} 13-\mathrm{H} 13$ & 108.2 \\
\hline $\mathrm{N} 1-\mathrm{C} 8-\mathrm{C} 1$ & $115.30(14)$ & $\mathrm{C} 14-\mathrm{C} 13-\mathrm{H} 13$ & 108.2 \\
\hline $\mathrm{S} 1-\mathrm{C} 9-\mathrm{N} 1$ & $123.45(12)$ & $\mathrm{C} 15-\mathrm{C} 13-\mathrm{H} 13$ & 108.2 \\
\hline $\mathrm{S} 1-\mathrm{C} 9-\mathrm{N} 2$ & $115.22(14)$ & $\mathrm{C} 13-\mathrm{C} 14-\mathrm{H} 141$ & 109.5 \\
\hline $\mathrm{N} 1-\mathrm{C} 9-\mathrm{N} 2$ & $121.32(15)$ & $\mathrm{C} 13-\mathrm{C} 14-\mathrm{H} 142$ & 109.5 \\
\hline $\mathrm{N} 2-\mathrm{C} 10-\mathrm{C} 11$ & $117.29(18)$ & $\mathrm{C} 13-\mathrm{C} 14-\mathrm{H} 143$ & 109.5 \\
\hline $\mathrm{S} 1-\mathrm{C} 11-\mathrm{C} 10$ & $108.91(16)$ & $\mathrm{H} 141-\mathrm{C} 14-\mathrm{H} 142$ & 109.5 \\
\hline $\mathrm{S} 1-\mathrm{C} 11-\mathrm{C} 12$ & $122.03(14)$ & $\mathrm{H} 141-\mathrm{C} 14-\mathrm{H} 143$ & 109.5 \\
\hline $\mathrm{C} 10-\mathrm{C} 11-\mathrm{C} 12$ & $129.06(19)$ & $\mathrm{H} 142-\mathrm{C} 14-\mathrm{H} 143$ & 109.5 \\
\hline $\mathrm{C} 1-\mathrm{C} 13-\mathrm{C} 14$ & $110.73(18)$ & C13-C15-H151 & 109.5 \\
\hline $\mathrm{C} 1-\mathrm{C} 13-\mathrm{C} 15$ & $111.30(18)$ & $\mathrm{C} 13-\mathrm{C} 15-\mathrm{H} 152$ & 109.5 \\
\hline $\mathrm{C} 14-\mathrm{C} 13-\mathrm{C} 15$ & $110.0(2)$ & $\mathrm{C} 13-\mathrm{C} 15-\mathrm{H} 153$ & 109.5 \\
\hline C8-N1-H111 & 118.3 & $\mathrm{H} 151-\mathrm{C} 15-\mathrm{H} 152$ & 109.5 \\
\hline $\mathrm{C} 9-\mathrm{N} 1-\mathrm{H} 111$ & 118.3 & $\mathrm{H} 151-\mathrm{C} 15-\mathrm{H} 153$ & 109.5 \\
\hline $\mathrm{C} 2-\mathrm{C} 1-\mathrm{H} 1$ & 107.7 & $\mathrm{H} 152-\mathrm{C} 15-\mathrm{H} 153$ & 109.5 \\
\hline $\mathrm{C} 8-\mathrm{C} 1-\mathrm{H} 1$ & 107.7 & & \\
\hline $\mathrm{C} 9-\mathrm{S} 1-\mathrm{C} 11-\mathrm{C} 10$ & $-0.73(16)$ & $\mathrm{C} 13-\mathrm{C} 1-\mathrm{C} 2-\mathrm{C} 7$ & $-65.9(2)$ \\
\hline $\mathrm{C} 9-\mathrm{S} 1-\mathrm{C} 11-\mathrm{C} 12$ & $178.9(2)$ & $\mathrm{C} 8-\mathrm{C} 1-\mathrm{C} 13-\mathrm{C} 14$ & $57.6(2)$ \\
\hline $\mathrm{C} 11-\mathrm{S} 1-\mathrm{C} 9-\mathrm{N} 1$ & $-178.04(16)$ & $\mathrm{C} 8-\mathrm{C} 1-\mathrm{C} 13-\mathrm{C} 15$ & $-179.67(16)$ \\
\hline $\mathrm{C} 11-\mathrm{S} 1-\mathrm{C} 9-\mathrm{N} 2$ & $0.70(15)$ & $\mathrm{C} 13-\mathrm{C} 1-\mathrm{C} 8-\mathrm{O} 1$ & $60.1(2)$ \\
\hline $\mathrm{C} 8-\mathrm{N} 1-\mathrm{C} 9-\mathrm{S} 1$ & $-1.7(2)$ & $\mathrm{C} 13-\mathrm{C} 1-\mathrm{C} 8-\mathrm{N} 1$ & $-118.44(17)$ \\
\hline $\mathrm{C} 8-\mathrm{N} 1-\mathrm{C} 9-\mathrm{N} 2$ & $179.66(17)$ & $\mathrm{C} 1-\mathrm{C} 2-\mathrm{C} 3-\mathrm{C} 4$ & $-178.9(2)$ \\
\hline $\mathrm{C} 9-\mathrm{N} 1-\mathrm{C} 8-\mathrm{O} 1$ & $-4.1(2)$ & $\mathrm{C} 1-\mathrm{C} 2-\mathrm{C} 7-\mathrm{C} 6$ & $-180.0(2)$ \\
\hline $\mathrm{C} 9-\mathrm{N} 1-\mathrm{C} 8-\mathrm{C} 1$ & $174.50(16)$ & $\mathrm{C} 3-\mathrm{C} 2-\mathrm{C} 7-\mathrm{C} 6$ & $0.7(3)$ \\
\hline $\mathrm{C} 9-\mathrm{N} 2-\mathrm{C} 10-\mathrm{C} 11$ & $-0.2(2)$ & $\mathrm{C} 7-\mathrm{C} 2-\mathrm{C} 3-\mathrm{C} 4$ & $0.4(3)$ \\
\hline $\mathrm{C} 10-\mathrm{N} 2-\mathrm{C} 9-\mathrm{S} 1$ & $-0.4(2)$ & $\mathrm{C} 2-\mathrm{C} 3-\mathrm{C} 4-\mathrm{C} 5$ & $-1.3(3)$ \\
\hline $\mathrm{C} 10-\mathrm{N} 2-\mathrm{C} 9-\mathrm{N} 1$ & $178.33(16)$ & $\mathrm{C} 3-\mathrm{C} 4-\mathrm{C} 5-\mathrm{Cl} 1$ & $-178.89(19)$ \\
\hline $\mathrm{C} 2-\mathrm{C} 1-\mathrm{C} 8-\mathrm{O} 1$ & $-65.9(2)$ & $\mathrm{C} 3-\mathrm{C} 4-\mathrm{C} 5-\mathrm{C} 6$ & $1.1(3)$ \\
\hline $\mathrm{C} 2-\mathrm{C} 1-\mathrm{C} 8-\mathrm{N} 1$ & $115.61(17)$ & $\mathrm{C} 11-\mathrm{C} 5-\mathrm{C} 6-\mathrm{C} 7$ & $179.97(13)$ \\
\hline $\mathrm{C} 8-\mathrm{C} 1-\mathrm{C} 2-\mathrm{C} 3$ & $-123.34(19)$ & $\mathrm{C} 4-\mathrm{C} 5-\mathrm{C} 6-\mathrm{C} 7$ & $-0.0(3)$ \\
\hline
\end{tabular}




\section{supporting information}

$\begin{array}{llll}\mathrm{C} 8-\mathrm{C} 1-\mathrm{C} 2-\mathrm{C} 7 & 57.4(2) & \mathrm{C} 5-\mathrm{C} 6-\mathrm{C} 7-\mathrm{C} 2 & -0.9(3) \\ \mathrm{C} 2-\mathrm{C} 1-\mathrm{C} 13-\mathrm{C} 14 & -178.17(17) & \mathrm{N} 2-\mathrm{C} 10-\mathrm{C} 11-\mathrm{S} 1 & 0.7(2) \\ \mathrm{C} 2-\mathrm{C} 1-\mathrm{C} 13-\mathrm{C} 15 & -55.5(2) & \mathrm{N} 2-\mathrm{C} 10-\mathrm{C} 11-\mathrm{C} 12 & -178.9(2) \\ \mathrm{C} 13-\mathrm{C} 1-\mathrm{C} 2-\mathrm{C} 3 & 113.3(2) & & \end{array}$

Hydrogen-bond geometry $\left(\AA,{ }^{\circ}\right)$

\begin{tabular}{lllll}
\hline$D-\mathrm{H} \cdots A$ & $D-\mathrm{H}$ & $\mathrm{H} \cdots A$ & $D \cdots A$ & $D-\mathrm{H} \cdots A$ \\
\hline $\mathrm{N} 1-\mathrm{H} 111 \cdots \mathrm{N} 2^{\mathrm{i}}$ & 0.86 & 2.08 & $2.929(2)$ & 168 \\
\hline
\end{tabular}

Symmetry code: (i) $-x+1, y,-z+3 / 2$. 